\title{
Crystalline Behavior of Paraffin Wax
}

Junfeng Wang, ${ }^{\text {a }}$ Yujian Hao, ${ }^{\mathrm{a}}$ Bojin Zhu, ${ }^{\mathrm{a}}$ Tiankun Han, ${ }^{\mathrm{a}}$ Zhen Li, ${ }^{\mathrm{a}}$ and Jun Zhang.*a

${ }^{\text {a }}$ School of Materials Science and Engineering, China University of Petroleum (East

China), Qingdao 266580, Shandong, China

Corresponding: zhangjun.upc@gmail.com 


\section{CONTENTS}

S1. Structure transformation of n-alkanes at $330 \mathrm{~K}$

S2. Distribution of n-alkanes in the crystal state .S6

S3. Dynamics details of paraffin wax at $330 \mathrm{~K}$

S4. Crystallization behavior of three systems with adding branched alkanes at $320 \mathrm{~K}$ .S8

S5. Pure $C_{30}$ n-alkane system at $320 \mathrm{~K}$ S9 


\section{S1. Structure transformation of n-alkanes at $330 \mathrm{~K}$}
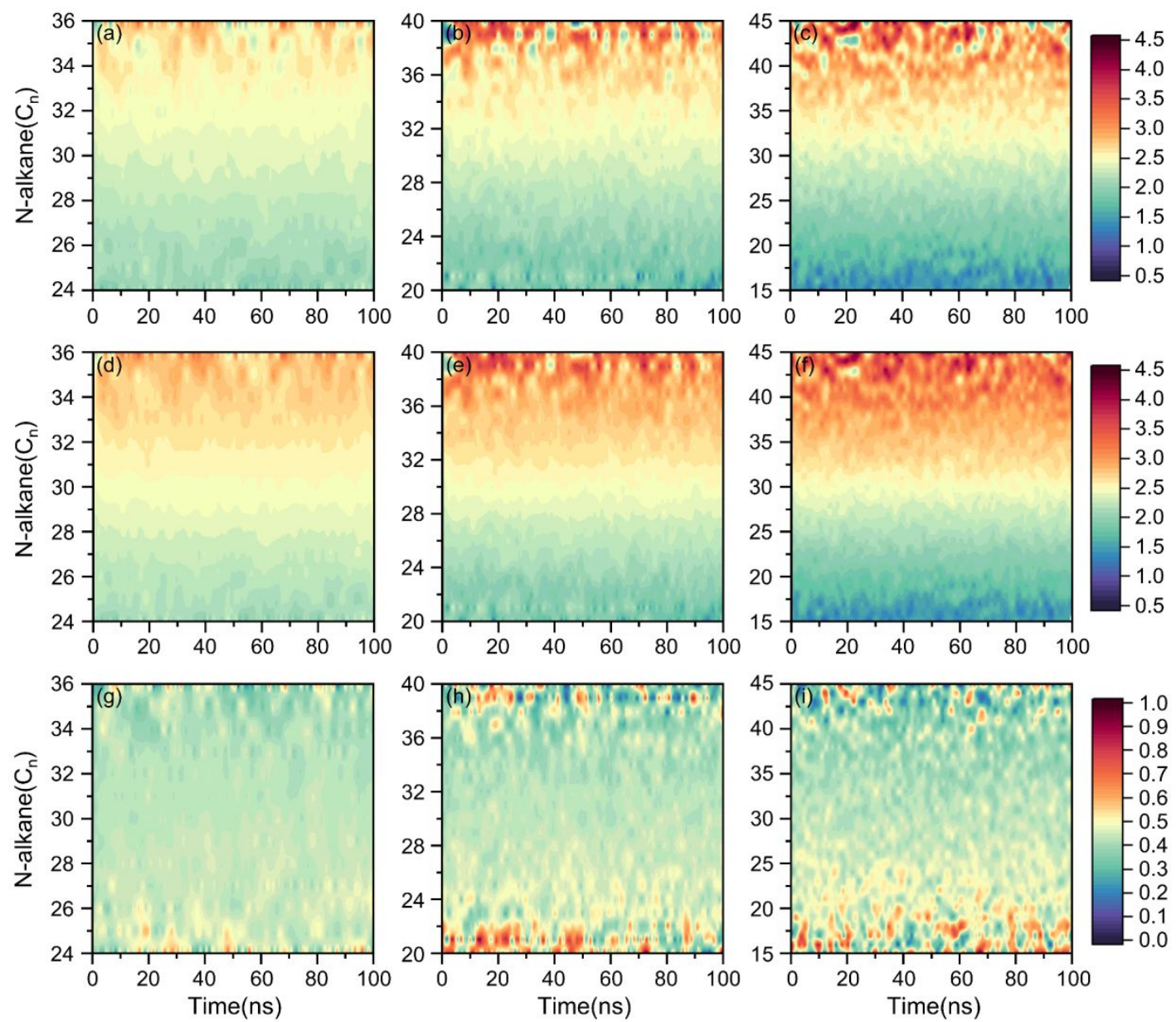

Figure S1. The average end-to-end distance $(\mathrm{Ed})$ of different n-alkanes $\left(\mathrm{C}_{\mathrm{n}}\right)$ over time in the 30-2 paraffin system (a), 30-3 paraffin system (b), and 30-5 paraffin system (c); The average diameter of the minimum envelope sphere $(\mathrm{Rg})$ of different n-alkanes $\left(\mathrm{C}_{n}\right)$ over time in the 30-2 paraffin system (d), 30-3 paraffin system (e), and 30-5 paraffin system (f); The average relative anisotropy $\left(\kappa^{2}\right)$ of different n-alkanes $\left(\mathrm{C}_{\mathrm{n}}\right)$ over time in the 30-2 paraffin system (g), 30-3 paraffin system (h), and 30-5 paraffin system (i). 

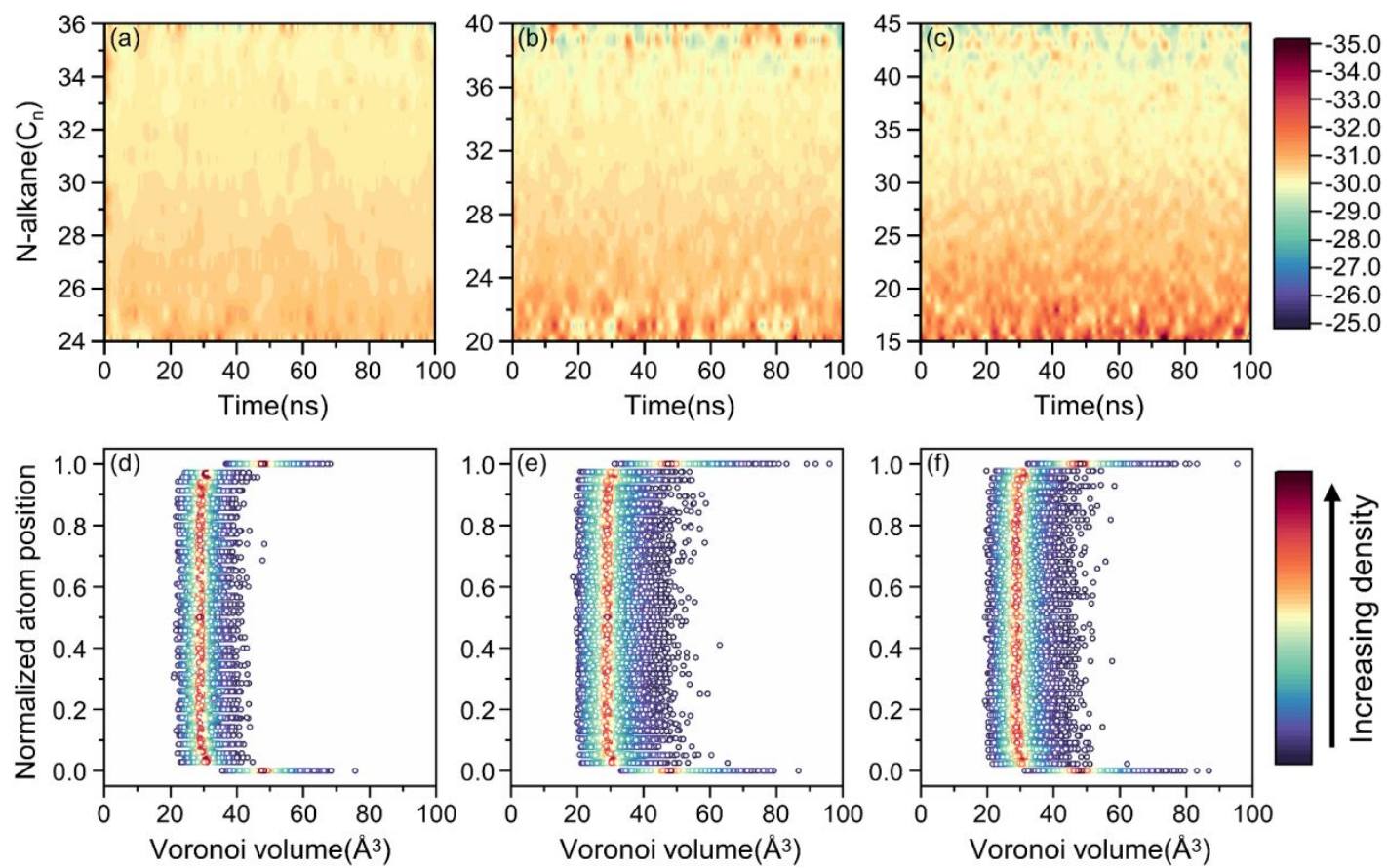

Figure S2. The average atomic Voronoi volume $\left(\overline{\mathrm{V}^{v}}\right)$ of every kind of different nalkanes $\left(\mathrm{C}_{n}\right)$ over time in the 30-2 paraffin system (a), 30-3 paraffin system (b), and 305 paraffin system $(\mathrm{c})$; All the Voronoi volume $\left(\mathrm{V}_{\mathrm{v}}\right)$ of every carbon atom in the whole simulation time in the 30-2 paraffin system (d), 30-3 paraffin system (e), and 30-5 paraffin system (f). 

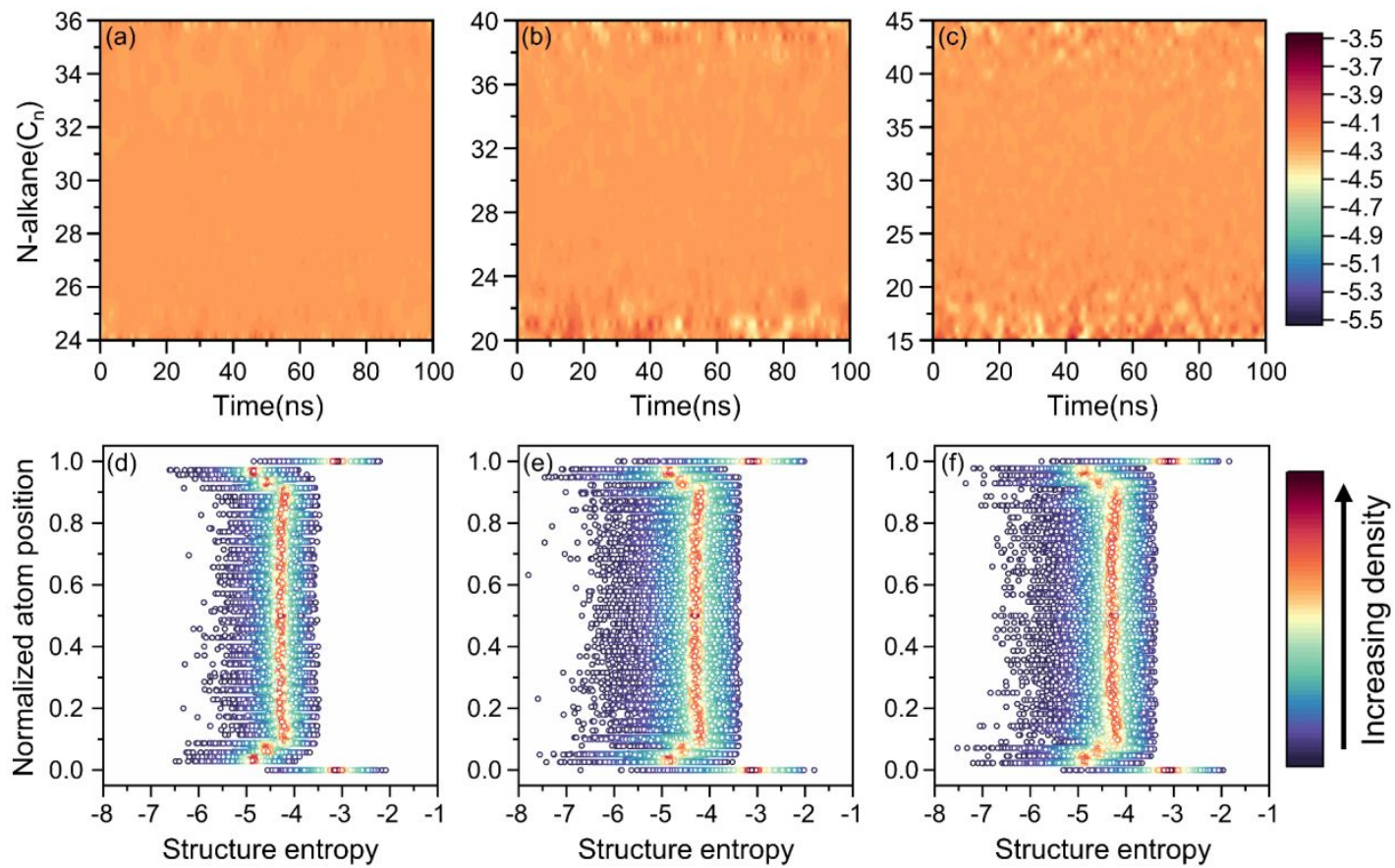

Figure S3. The average atomic structure entropy $\left(\overline{\mathrm{S}^{2}}\right)$ of different n-alkanes $\left(\mathrm{C}_{\mathrm{n}}\right)$ over time in the 30-2 paraffin system (a), 30-3 paraffin system (b), and 30-5 paraffin system (c); All the structure entropy $\left(\mathrm{S}_{2}\right)$ of every carbon atom in the whole simulation time in the 30-2 paraffin system (d), 30-3 paraffin system (e), and 30-5 paraffin system (f). 
S2. Distribution of $\mathbf{n}$-alkanes in the crystal state

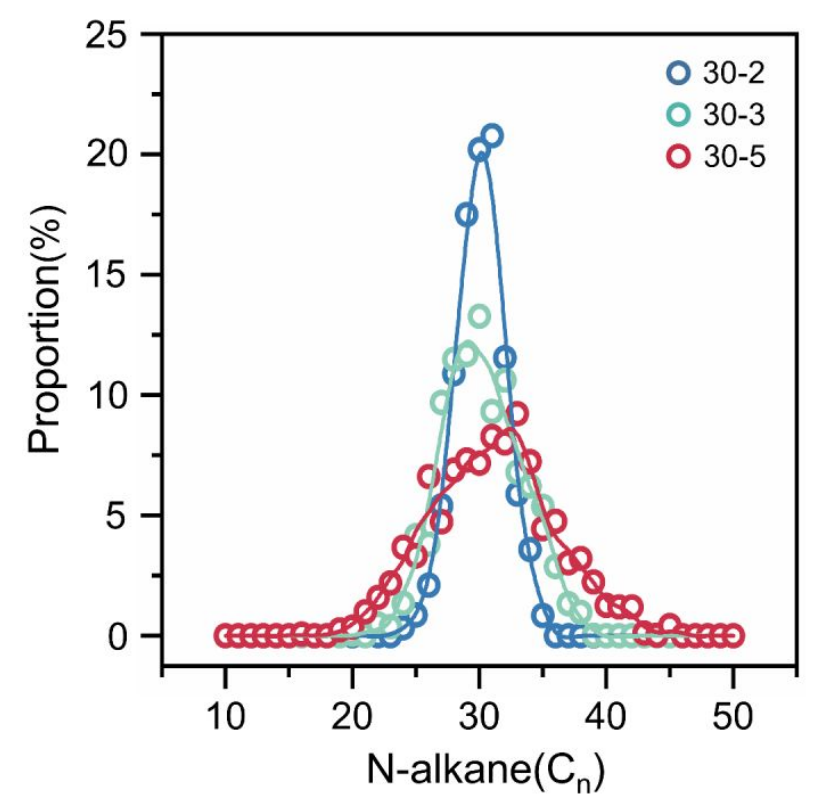

Figure S4. The proportion of different n-alkanes $\left(C_{n}\right)$ in the crystal state. 


\section{S3. Dynamics details of paraffin wax at $330 \mathrm{~K}$}
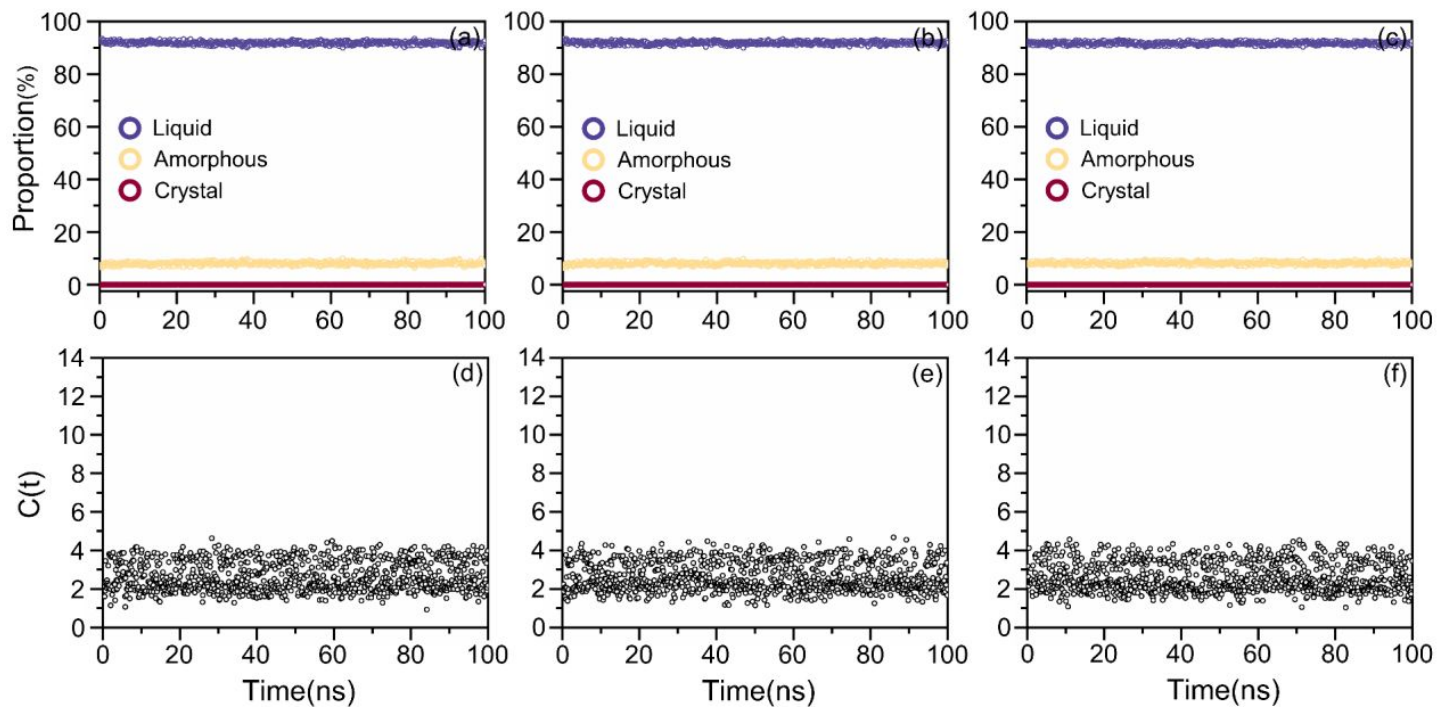

Figure S5. The proportion of three phase states over time in the 30-2 paraffin system (a), 30-3 paraffin system (b), and 30-5 paraffin system (c); The density correlation function $C(t)$ over time in the 30-2 paraffin system (d), 30-3 paraffin system (e), and 30-5 paraffin system (f).

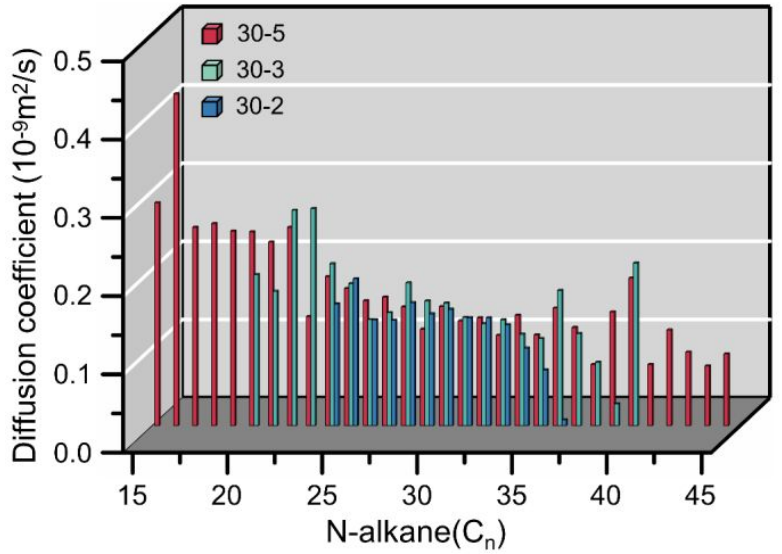

Figure S6. The average diffusion coefficient of different n-alkanes $\left(\mathrm{C}_{n}\right)$ to three paraffin systems at $330 \mathrm{~K}$. 


\section{S4. Crystallization behavior of three systems with adding branched}

\section{alkanes at $320 \mathrm{~K}$}
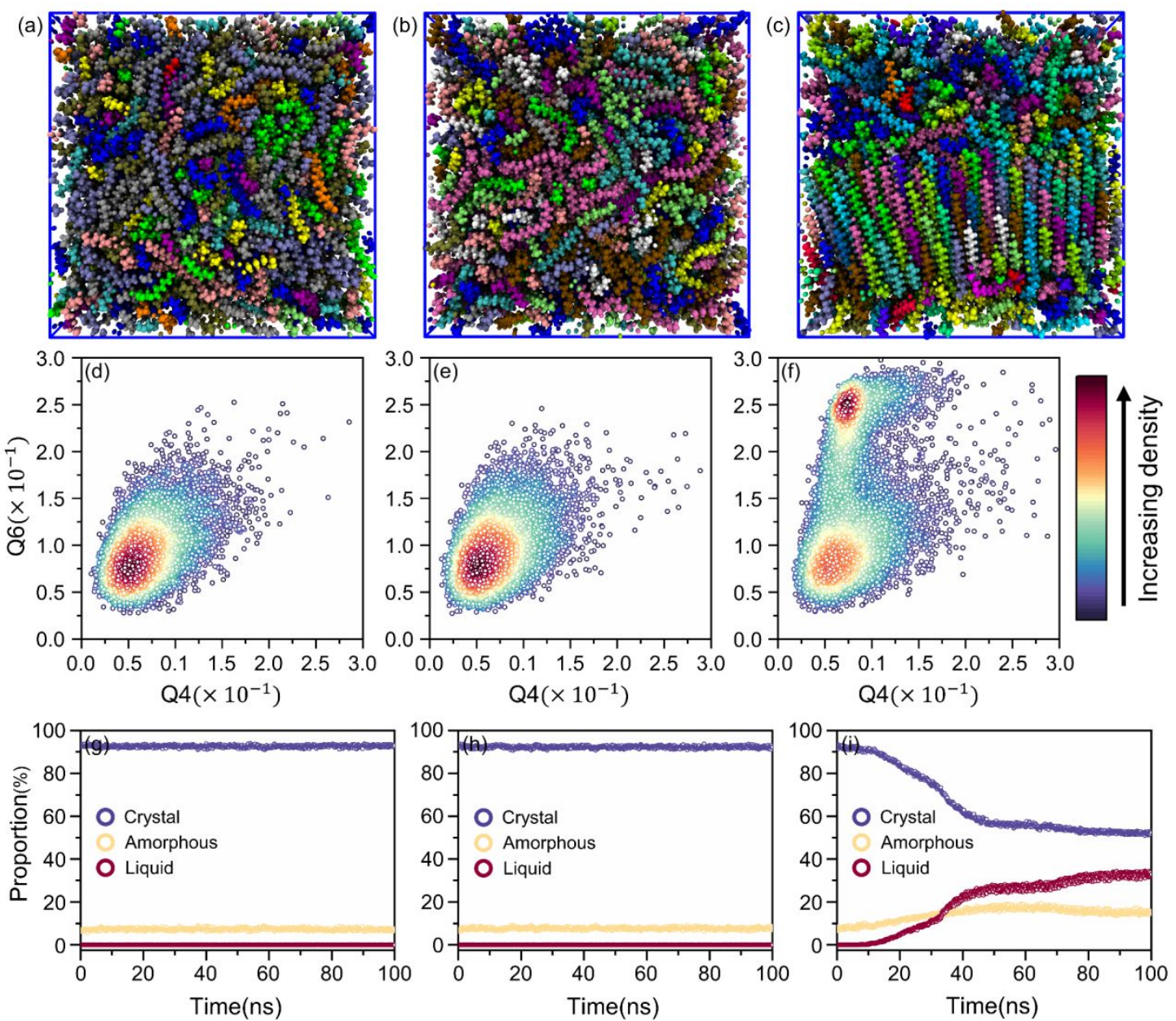

Figure S7. The final morphology of the 30-2 paraffin system (a), 30-3 paraffin system (b), and 30-5 paraffin system (c) when half of the $\mathrm{C}_{30}$ n-alkane is changed into the perhydrosqualene $\left(\mathrm{P}_{30}\right)$, and the proportion of $\mathrm{P}_{30}$ is $10 \%(\mathrm{a}), 6.7 \%(\mathrm{~b}), 4 \%$ (c); The order parameters Q4 and Q6 of every carbon atom after 100ns in the 30-2 paraffin system (d), 30-3 paraffin system (e), and 30-5 paraffin system (f); The proportion of three phase states over time in the 30-2 paraffin system (g), 30-3 paraffin system (h), and 30-5 paraffin system (i). 
S5. Pure $\mathrm{C}_{30}$ n-alkane system at $320 \mathrm{~K}$
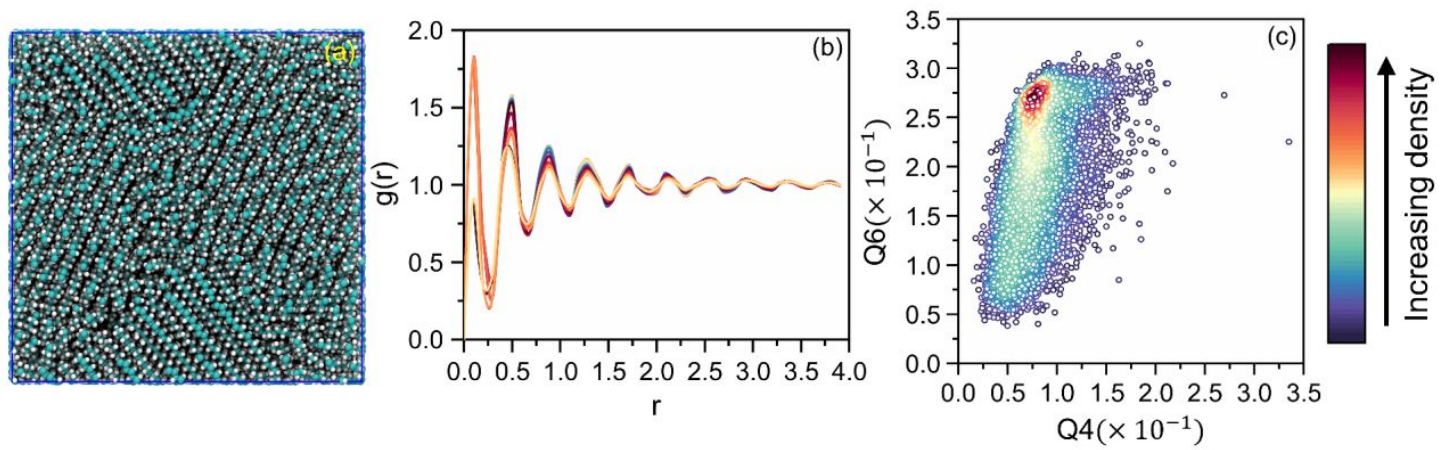

Figure S8. (a) The final morphology of the $C_{30}$ n-alkane system. (b) The average radial distribution function of the carbon atoms with different positions in the n-alkane molecule. (c) The order parameters Q4 and Q6 of every carbon atom after $100 \mathrm{~ns}$. 